\section{or 不健康素食饮食可能伤肾}

有观点认为吃素能够起到保护肾脏、降低肾病风险的 作用, 但一项新研究指出, 吃素也有讲究, 常吃富含淀粉 和糖的素食反而可能会增加肾脏损伤的风险。

《美国肾病学会临床杂志》网络版近日刊载的一项研 究说, 约翰斯 - 霍普金斯大学等机构研究人员分析了 1.4 万多名成年人的饮食习惯和肾功能状况, 并对其中一半人 开展 24 年以上的追踪调查。总计 4343 人在研究期间患上 慢性肾病。

研究发现, 与很少吃健康素食的人相比, 坚持健康素 食的人患肾病风险要低 $14 \%$; 与很少吃不健康素食的人相 比, 常吃不健康素食的人患肾病风险会高出 $11 \%$ 。

来源: 环球网

愉快心情吃出来! 英国研究发现饮食均衡有利 于缓解抑郁

你是否时常感觉闷闪不乐、意志消沉? 你有没有想过 这种症状可以通过改善饮食习惯来治愈? 据法国 LCI 电 视台报道, 英国曼彻斯特大学研究人员近日在美国《身 心医学》杂志上发表的一篇报告表示, 饮食健康均衡有 助于缓解抑郁症的症状, 并且这种影响在女性身上更为 明显。

研究人员对 4.6 万名疑似患有非临床抑郁症的受调查 者进行分析, 结果发现优化饮食习惯可以减轻抑郁症状。 三种常见的饮食干预都有类似的积极效果: 减肥、减少脂 肪摄入以及增加饮食中的营养摄入。

参与该研究夫人约瑟夫 - 弗思 (Joseph Firth) 博士说: “饮食对心理健康影响的总体证据还有待评估。但是我们 最新的分析表明, 采取更健康的饮食可以改善人们的情 绪。”

$$
\text { 来源: 环球网 }
$$

\section{英媒: 全球酒精消费量显著增加部分归因于} 中国

据英国《卫报》5 月 8 日报道, 研究人员称, 与 30 年 前相比, 全球的酒精消费量显著增加, 这在很大程度上要 归因于中国和印度酗酒和大范围饮酒的现象日益增加。

据研究人员在《柳叶刀》杂志上的报道, 按照目前的
趋势, 全球人均酒精消费量在 1990 年至 2017 年间增长了 $10 \%$, 未来 10 年将再增长 $17 \%$ 。通过调查 189 个国家, 该调查的预测结果显示, 到 2030 年, 全球超过一半的成 年人将饮酒, 近四分之一的人将至少每月狂饮一次。

全球约有 2.37 亿男性和 4600 万女性患有与酒精有关 的疾病, 其中欧洲和北美的比例最高。

$$
\text { 来源: 环球网 }
$$

研究: 怀孕早期吃坚果能提高孩子智力

据英国《每日电讯报》5 月7 日报道, 一项新的研究表明, 在怀孕早期经常吃坚果的准妈妈, 孩子会更加聪明。

研究者对 2200 名妇女及其子女进行了长达八年以上 研究, 分析发现, 在怀孕初期的 12 周内, 每周吃三份或 三份以上坚果的母亲, 她们的孩子在智力测试中表现更好。 这些孩子的认知功能、注意力广度和工作记忆都优于那些 母亲在怀孕前三个月很少或根本不吃坚果的孩子。

西班牙巴塞罗那全球健康研究所的研究小组认为, 在 至关重要的早期发育阶段, 叶酸和必需脂肪酸 (如 omega-3 和 omega-6) 等有益的营养物质在发育中的胎儿的神 经组织中积累。他们还探索了最后三个月的智力和高坚果 摄入量之间的联系, 但并未发现有任何联系。

$$
\text { 来源: 环球网 }
$$

\section{“脱欧”致使英国护士及助产士进一步短缺}

据英国《卫报》5 月 8 日报道, 过去两年中, 来自欧 盟 27 个国家的近 5000 名护士和助产士退出了英国国家医 疗服务体系 (NHS), 其中许多人认为这是由脱欧导致的。

英国 “护理及助产委员会” (NMC) 负责管控这两项 职业。该机构的数据表明, 欧盟培训的护士和助产士在英 国全国卫生服务机构工作的人数从 2017 年 3 月的 3.8 万余 人的创纪录峰值下降到了今年 3 月的 3.3 万人, 共减少了 4989 人。此次 $13 \%$ 的降幅再次引起警惕, 人们认为英国 决定脱欧加剧了英国国家医疗服务体系不断恶化的人员短 缺危机。

英国工会联合会的卫生主管萨拉・戈顿说: “许多欧 盟成员国的国民不赞成这次公投结果。护士和助产士也认 为他们会在其他地方获得更好的待遇, 这很正常。” 